\title{
Malignant transformation of tracheal inflammatory pseudotumor: A case report
}

\author{
Sayyed Abbas Tabatabaee, MD, ${ }^{\mathrm{a}}$ Sayyed Mozafar Hashemi, MD, ${ }^{\mathrm{a}}$ Mojtaba Ahmadi Nejad, MD, ${ }^{\mathrm{b}}$ Amir Hosein Davarpanah Jazi, \\ Mohammad Eidy, MD, ${ }^{\mathrm{d}}$ Parvin Mahzouni, MD, ${ }^{\mathrm{e}}$ and Ali Hekmatnia, MD, ${ }^{\mathrm{f}}$ Isfahan and Khorram Abad, Iran
}

Inflammatory pseudotumors (IPTs) are uncommon masses in the lung parenchyma, pleura, or bronchi, and they represent less than $1 \%$ of all tumors in the lungs and airways. ${ }^{1}$ The reported intratracheal IPTs to date are fewer than 15 cases, and all were benign. These tumors are most commonly found in the lung and orbit. These lesions are rare, but they are the most common primary lung neoplasms in the pediatric population. The majority of these pseudotumors are located in the lung parenchyma, but $5 \%$ to $16 \%$ are endobronchial. ${ }^{2}$ Although these lesions commonly have benign characteristics, distant metastasis and sarcomatous transformation have been rarely reported. ${ }^{3-6}$ We found no reported case of malignant IPT of the trachea in the published English literature and this is the first report of tracheal IPT.

\section{CLINICAL SUMMARY}

In November 2006, a 13-year-old boy was referred to our hospital with dyspnea and diffuse wheezing of more than 19 months' duration. The symptoms had started after aspiration of stomach contents following difficulty in respiration and decreased consciousness in June 2005. Three months later, he was brought to the emergency department with severe dyspnea, which led to respiratory failure and admission to the intensive care unit. One week later, his condition was stable. On the basis of clinical manifestations and a restrictive pattern of spirometry (forced vital capacity (FVC) = $85.8 \%$, forced expiratory volume in 1 second $\left(\mathrm{FEV}_{1}\right)=$ $48.4 \%$, and $\mathrm{FEV}_{1} / \mathrm{FVC}=47 \%$ ), a diagnosis of severe asthma was made and treatment was started with beta agonists and corticosteroids. Despite use of full-dose inhalations for 13 months, not only did the patient's symptoms fail to improve, but also cough was added to the picture. Gradually, deterioration of the patient's signs and symptoms led to further evaluations. In October 2006, a chest radiograph showed left lung atelectasis. A postcontrast computed tomographic scan of the chest demonstrated a soft tissue mass of $3 \times 2.5 \times 2 \mathrm{~cm}$ within the trachea, $2 \mathrm{~cm}$ above the carina, which had created

\footnotetext{
From the Department of Thoracic Surgery, AL Zahra Hospital, Isfahan University of Medical Science (IUMS), Isfahan, Iran, ${ }^{a}$ the Department of Surgery, Shohada Hospital, Lorestan University of Medical Science, Khorram Abad, Iran, ${ }^{\mathrm{b}}$ the Talent Development Office, IUMS, ${ }^{\mathrm{c}}$ and the Departments of General Surgery, ${ }^{\mathrm{d}}$ Pathology, and Radiology, ${ }^{\mathrm{f}}$ AL Zahra Hospital, IUMS, Isfahan, Iran.

Received for publication Nov 12, 2007; accepted for publication Dec 16, 2007.

Address for reprints: Amir Hosein Davarpanah Jazi, Education and Development Center (EDC), room No. 103, Isfahan University of Medical Science, Hezar Jereeb St, Isfahan, 80748673441, Iran (Post box: 353- 81745) (E-mail: davarpanah@edc. mui.ac.ir)

J Thorac Cardiovasc Surg 2009;137:747-9

$0022-5223 / \$ 36.00$

Copyright (c) 2009 by The American Association for Thoracic Surgery doi:10.1016/j.jtcvs.2007.12.073
}

marked narrowing of the airway (Figure $1, A$ ). The patient was hospitalized and laboratory tests on admission were as follows: white blood cell count, $5420 / \mathrm{mL}$; red blood cell count, 4.52 million/mL; hemoglobin, $13.7 \mathrm{~g} / \mathrm{dL}$; hematocrit, $38.3 \%$; mean cell volume, $84.7 \mathrm{fl}$; mean corpuscular hemoglobin, $30.33 \mathrm{pg} / \mathrm{red}$ cell; platelet count, 278,000/mL; blood urea nitrogen, $12 \mathrm{mg} / \mathrm{dL}$; creatine, $0.7 \mathrm{mg} / \mathrm{dL}$; sodium, 136 $\mathrm{mEq} / \mathrm{mL}$; and potassium, $3.7 \mathrm{mEq} / \mathrm{mL}$.

During examination with a rigid bronchoscope, a round, smooth, pink pedunculated mass adhering to the left tracheal wall, $2 \mathrm{~cm}$ above the carina, was seen. The tumor was totally removed with forceps, and the mucosa of the insert point of the tumor pedicle in the bronchus was electrocoagulated without any bleeding complication. Pathologic examination of the mass revealed a loose stroma with chronic infiltration of histiocytic lymphoplasma cells, vascular proliferation, fibrosis, hyalinization, partial myxoid changes, and marginal cogwheel nuclei (Figure 2, A). In immunohistochemical (IHC) studies, spindle cells were positive for vimentin; background lymphocytes stained by leukocyte common antigen but they were negative for actin, desmin, and S100. Therefore, diagnosis of IPT was made on the basis of histopathologic features of the tumor.

Five months later, he returned with cough and dyspnea without weight loss or hemoptysis. Chest radiographs as well as chest computed tomographic scan demonstrated involvement of the distal part of the trachea whereas the proximal part of the left main bronchus was intact. Moreover, a round extratracheal soft tissue mass located in close proximity to the posterior tracheal wall was seen (Figure 1, B). The patient was hospitalized and the second tracheobronchoscopy with a rigid endoscope was performed under general anesthesia. This study showed a polypoid fragile mass with a thick edematous irregular mucosal surface starting from $4.5 \mathrm{~cm}$ above the carina and extending $2.5 \mathrm{~cm}$ distally. Histopathologic examination revealed a lesion composed of an admixture of spindle cells with hyperchromic angulated nuclei and cellular pleomorphism sometimes dispersed in herringbone configuration and chronic inflammatory cells including plasma cells, lymphocytes, eosinophils and macrophages. Areas of necrosis were also present (Figure 2, B). In IHC studies, spindle cells were positive for vimentin, p53, leukocyte common antigen, and weakly focally for smooth muscle actin, but were negative for S-100, desmin, and cytokeratin. Findings were consistent with a diagnosis of IPT with malignant transformation.

Two weeks later, he was taken to the operating room. Thoracotomy showed extratracheal extension of the tumor 


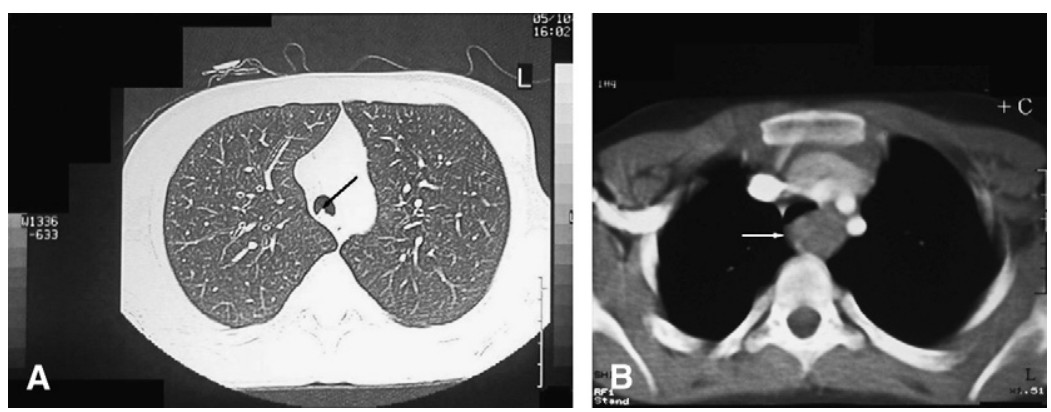

FIGURE 1. Computed tomographic scans. A, The arrow shows intratracheal lobulated soft tissue lesion. B, Five months later, a lobulated soft tissue lesion in the posterior aspect of the trachea with prominent extratracheal extension was observed (arrow).

and involvement of the loose connective tissue surrounding the trachea. However, the inferior vena cava, aortic arch, and esophagus were intact. The involved area of the trachea with a $10-\mathrm{mm}$ safe margin was successfully excised. The right pulmonary hilum was completely released through pericardium. The trachea was mobilized and the continuity of upper airways was obtained with such a procedure. Both light microscopy and IHC confirmed the malignant transformation of IPT. During a follow-up period of 8 months, there was no recurrence or distal metastasis.

\section{DISCUSSION}

IPTs are generally benign lesions that most commonly arise from the lung but may involve nearly all sites of the body. Despite predominant involvement of pulmonary parenchyma, tracheal IPTs are uncommon. The etiology of this tumor is unclear. Some investigators believe that this tumor develops as part of a nonspecific inflammatory reaction resulting from trauma, autoimmune reaction, or infection. Histologically, IPT is characterized by benign spindle cell proliferation in a myxoid and vascular stroma including inflammatory components of lymphocytes, plasma cells, eosinophils, foamy macrophages, neutrophils, and histiocytes. Given their possible local invasiveness, tendency to recur, and marked cellularity, these tumors may be mistaken even by experienced pathologists for neoplasms such as spindle cell carcinoma, eosinophilic granuloma, fibrosarcoma, rhabdomyosarcoma, infantile myofibromatosis, subtle melanoma, and possibly Hodgkin's lymphoma. Difficult cases such as infantile myofibromatosis and rhabdomyosarcoma can be excluded using IHC stains with careful morphologic assessment. Cytokeratin stain, S-100 stain, and HMB45 stain are negative in IPTs. ${ }^{3}$ The local recurrences, distant metastases, clonal chromosomal aberrations, and even sarcomatous progression described in a few reported cases suggest the malignant potential of IPTs. ${ }^{5}$ Detection of P53 expression by IHC could differentiate malignant neoplasm from benign lesion. In the presented case, the demonstration of a p53 mutation clearly established the neoplastic nature of the tumor and distinguished it from any nonneoplastic pseudotumor. Conservative surgery is the current treatment for IPT. Local removal with a margin of normal tissue should be performed to lower the possibility of local recurrence. Coffin and associates $^{7}$ found a recurrence rate of $25 \%$ for incomplete resection. Overall, aggressive behavior of the tumor is associated with the tumor anatomic site and its proximity to vital structures, which compromise definitive resection. Radiotherapy should be reserved as a choice treatment when surgical
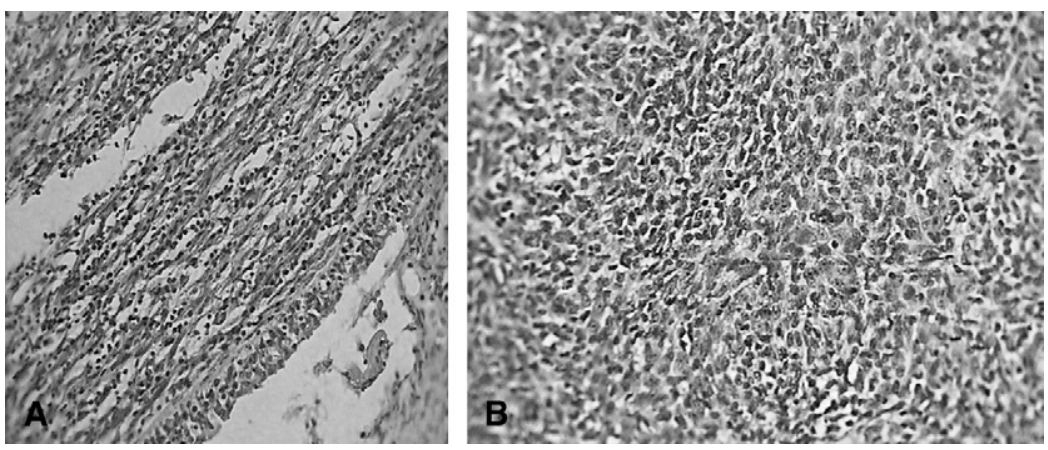

FIGURE 2. Photomicrographs of tracheal specimen. A, Endobronchial polypoid mass with spindle cells in background of inflammatory cells including plasma cells, lymphocytes, and histiocytes. This slide was prepared on October 2006 (hematoxylin and eosin; original magnification $\times 40$ ). B, Five months later, hypercellularity and mitosis were seen in the photomicrographs (hematoxylin and eosin; original magnification $\times 40$ ). Necrosis was present at another area of the slide, which is not shown here. 
intervention is impossible. Chemotherapy has shown no beneficial outcome unless malignancy is present. The overall prognosis for IPT is excellent. Most recurrences will arise during the first year after initial therapy. Only a few reports showed late-onset recurrences.

\section{References}

1. Dunnick NR. Image interpretation session: 1999. Inflammatory myoblastic pseudotumor of the trachea (plasma cell granuloma). Radiographics. 2000;20:274-6.

2. Cerfolio RJ, Matthews TC. Resection of the entire left mainstem bronchus for an inflammatory pseudotumor. Ann Thorac Surg. 2005;79:2127-8.
3. Amir R, Danahey D, Ferrer K, Maffee M. Inflammatory myofibroblastic tumor presenting with tracheal obstruction in a pregnant woman. Am J Otolaryngol. 2002;23:362-7.

4. Browne M, Abramson LP, Chou PM, Acton R, Holinger LD, Reynolds M. Inflammatory myofibroblastic tumor (inflammatory pseudotumor) of the neck infiltrating the trachea. J Pediatr Surg. 2004;39:e1-4.

5. Bumber Z, Jurlina M, Manojlovic S, Jakic-Razumovic J. Inflammatory pseudotumor of the trachea. J Pediatr Surg. 2001;36:631-4.

6. Nikanne E, Sopanen J, Seppa A. Inflammatory pseudotumor of the trachea. Otolaryngol Head Neck Surg. 2004;130:274-6.

7. Coffin CM, Watterson J, Priest JR, Dehner LP. Extrapulmonary inflammatory myofibroblastic tumor (inflammatory pseudotumor). A clinicopathologic and immunohistochemical study of 84 cases. Am J Surg Pathol. 1995;19: 859-72.

\section{Infective mitral valve myxoma with coronary artery embolization: Surgical intervention followed by prolonged survival}

Feng Yao, MD, Zhi-yun Xu, MD, Yan-lin Liu, MS, and Lin Han, MD, Shanghai, People's Republic of China

Three-quarters of primary cardiac neoplasms are benign; nearly half of benign heart tumors are myxomas, which have an estimated incidence of 0.5 per million population per year. ${ }^{1,2}$ Roughly $75 \%$ of myxomas originate from the left atrium, and $15 \%$ to $20 \%$ originate from the right atrium. Myxomas of the heart valves are exceedingly rare. ${ }^{1}$ Myxomas may simulate infective endocarditis, but rarely are they actually infected.

We report a case of a 12-year-old child with an infective myxoma originating from the ventricular side of the anterior mitral leaflet with a coronary artery embolism.

\section{CLINICAL SUMMARY}

A 12-year-old girl from a rural area of mainland China with a 9-year history of recurrent intermittent fevers and night sweats was admitted to our hospital on April 4, 2001. During the 9 years prior to admission, the girl was diagnosed with an upper respiratory tract infection each time she developed high fever. This diagnosis was given because no other positive evidence could be found, and the girl was treated with empirical antibiotics. At the time of admission,

From the Department of Cardiothoracic Surgery, Changhai Hospital, Second Military Medical University, Shanghai, People's Republic of China.

Received for publication Nov 24, 2007; revisions received Jan 8, 2008; accepted for publication Jan 15, 2008.

Address for reprints: Zhi-yun Xu, MD, Department of Cardiothoracic Surgery, Changhai Hospital, 174 Changhai Road, Shanghai 200433, People's Republic of China (E-mail: zhiyunx@hotmail.com).

J Thorac Cardiovasc Surg 2009;137:749-51

$0022-5223 / \$ 36.00$

Copyright (C) 2009 by The American Association for Thoracic Surgery

doi:10.1016/j.jtcvs.2008.01.050 however, she had maintained fevers as high as $103.5^{\circ} \mathrm{F}$ $\left(39.5^{\circ} \mathrm{C}\right)$ for 3 weeks. These fevers were unresponsive to high doses of intravenous penicillin. Moreover, the patient had developed severe dyspnea and lower extremity edema. There was a III/6 soft, blowing, systolic murmur at the apex on auscultation.

Neisseria lactamica was isolated from blood cultures on 2 consecutive days, and an emergency echocardiogram was obtained, which revealed a mass in the left ventricle attached to the anterior mitral leaflet, with severe mitral regurgitation (Figure 1).

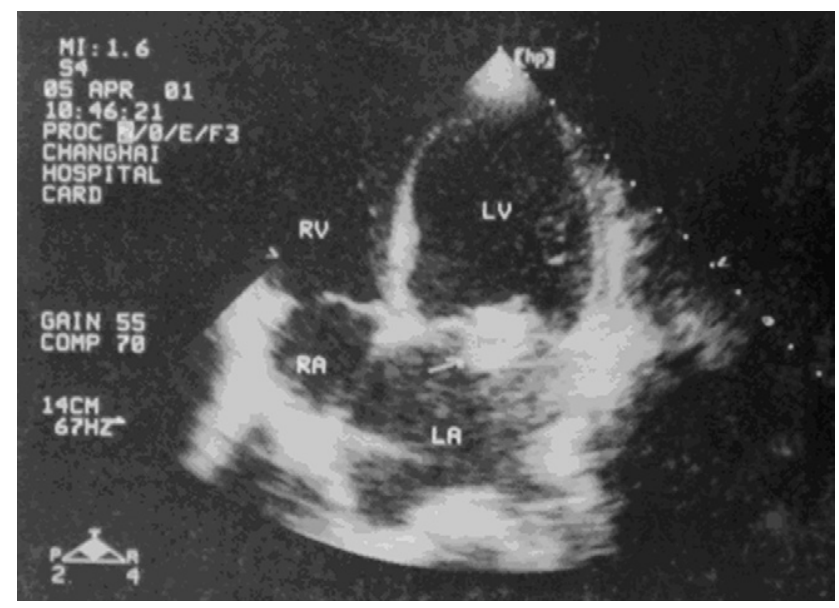

FIGURE 1. Transthoracic echocardiography in the 4-chamber apical view, revealing a left ventricular mass (arrow) attached to the anterior mitral leaflet. $L V$, left ventricle; $R V$, right ventricle; $L A$, left atrium; $R A$, right atrium. 